\title{
RDUS
}

Revue de DROIT

UNIVERSITÉ DE SHERBROOKE

Titre : $\quad$ LE FORMULAIRE DE CONSENTEMENT À LA RECHERCHE :

INCOMPATIBILITÉ ENTRE LE DROIT DES PERSONNES ET LE DROIT DES CONTRATS

Auteur(s): $\quad$ Julien P. CABANAC

Michel T. GIROUX

Revue : $\quad$ RDUS, 2006-2007, volume 37, numéro 2

Pages: $\quad 235-274$

ISSN : $\quad$ 0317-9656

Éditeur : $\quad$ Université de Sherbrooke. Faculté de droit.

URI : $\quad$ http://hdl.handle.net/11143/11836

DOI : $\quad$ https://doi.org/10.17118/11143/11836 
Page vide laissée intentionnellement. 


\title{
ARTICLE
}

\section{LE FORMULAIRE DE CONSENTEMENT À LA RECHERCHE : INCOMPATIBILITÉ ENTRE LE DROIT DES PERSONNES ET LE DROIT DES CONTRATS ${ }^{*}$}

\begin{abstract}
par Julien P. CABANAC ${ }^{* *}$ Michel T. GIROUX ${ }^{* * *}$

Le formulaire de consentement est un document que les sujets de recherche signent afin d'attester qu'ils acceptent de se prêter à un projet de recherche. Ce document expose l'objet de la recherche, la participation du sujet pressenti et, surtout, les risques auxquels celui-ci s'expose. Or, le formulaire de consentement est souvent ardu à lire et il contient une quantité telle d'informations que l'on peut douter de l'authenticité du consentement exprimé. Comment expliquer que le formulaire de consentement devienne de plus en plus détaillé et compliqué? Nous avançons que ce document est rédigé dans un contexte légal qui favorise cette évolution. En droit civil québécois, la forme écrite du consentement à la recherche s'inscrit dans deux cadres faisant appel à des rationalités différentes: le droit des personnes et le droit des contrats. En droit contractuel, l'approche générale veut que l'écrit représente l'entente intervenue entre les contractants de la façon la plus complète et précise possible. Il n'existe pas de limite juridique à la longueur des contrats. En revanche, l'éthique de la recherche et le droit des personnes attribuent une importance décisive à l'authenticité du consentement du sujet. Le respect des droits des sujets requiert que les responsables de la recherche en santé, notamment les comités d'éthique de la recherche (CÉR), soient attentifs à ce que le caractère contractuel du formulaire n'engendre pas une réduction de la qualité ou de l'authenticité du consentement. Considérant que la réception d'une grande quantité d'informations risque de nuire à la qualité du consentement, les chercheurs et les CÉR devraient restreindre la longueur des formulaires de consentement à quelques pages, c'est-à-dire à l'essentiel des informations utiles.
\end{abstract}

* . Les opinions émises dans cet article ne lient que les auteurs.

** . B.Sc., Ll.M, Ll.D., avocat chez Paul-Claude BÉRubé, Avocats, à SaintJean-sur-Richelieu.

*** Avocat, docteur en philosophie, directeur de l'Institut de consultation et de recherche en éthique et en droit (ICRED). 
Le formulaire de consentement à la recherche : incompatibilité entre (2007) 37 R.D.U.S. le droit des personnes et le droit des contrats

Consent forms are documents which research subjects sign, attesting to the fact that they accept to participate in a research project. This document explains the purpose of the research, the willingness to participate of the prospective subject and underlines the potential risks to which the person is exposed. Consent forms are frequently difficult to comprehend and often contain a plethora of detail which may affect the validity of the consent given. Why are consent forms becoming so detailed and complicated? The writers posit that the present legal climate lends itself to this evolution. In the Civil Code of Buebec, the requirement of written consent pertains to two different contexts; human rights law and contract law. In contract law, it is generally understood that a written document represents the agreement between the contracting parties and should thus be as complete as possible. There are no legal restrictions on the actual length of the contract. Yet, research ethics and human rights considerations attach great importance to the genuineness of the subject's consent. Respecting the rights of research subjects requires that those in charge of health research, more particularly research ethics boards (REBs), be attentive to the fact that the contractual nature of consent forms must not compromise the authenticity of the consent given. Since information overload may affect the actual quality of consent, researchers and REBs should limit the length of consent forms to a few pages, that is to say, to containing that information which is deemed most useful. 


\section{SOMMAIRE}

Introduction

1. La présence d'un écrit au contenu toujours plus détaillé

2. Pourquoi l'écrit est-il source de confusion?................ 245

a) La protection du sujet de recherche.............. 245

b) La preuve écrite du consentement ................250

c) Le droit des personnes et le droit des contrats ............................................... 254

B. Le droit des personnes et le droit des contrats sont-ils des vases communicants .................................... 256

1. L'indisponibilité de la personne et le commerce ..........258

2. Une liberté contractuelle limitée ........................... 263

3. Le formulaire conçu comme un contrat : conséquences pratiques .................................. 266

Conclusion $\quad$.......................................................... 272 

Le formulaire de consentement

(2007) 37 R.D.U.S. à la recherche : incompatibilité entre

le droit des personnes et le droit des contrats

\section{Introduction}

Depuis plus d'un demi-siècle, l'éthique de la recherche en santé a développé des règles dans le but de protéger les participants contre les risques de l'expérimentation. Des questionnements sur la légitimité de la participation des sujets de recherche se trouvent à l'origine de considérations éthiques importantes concernant l'exercice de l'autodétermination personnelle. Il est clair aujourd'hui que le respect essentiel des personnes ${ }^{1}$ impose aux chercheurs d'obtenir le consentement des sujets pressentis avant de les recruter à titre de participants.

Au Canada, la règle générale prévoit que la participation des sujets de recherche requiert la signature préalable d'un formulaire de consentement ${ }^{2}$. Dans le cas de la recherche commanditée, le formulaire fait partie intégrante d'un projet rédigé par le commanditaire ou par le promoteur de la recherche. Le plus souvent, le commanditaire est une compagnie pharmaceutique qui cherche à développer un nouveau médicament. Après avoir monté son projet, celui-ci contacte des chercheurs potentiels (usuellement, des médecins) de plusieurs établissements afin qu'ils acceptent de mettre en œuvre son projet de recherche. Avant d'entreprendre la réalisation de leur projet, les chercheurs doivent recevoir l'approbation du comité d'éthique de la recherche (CÉR) de chaque institution qui accueillera le projet. Le projet de

1. É.-U., National Commission for the Protection of Human Subjects of Biomedical and Behavioral Research, Rapport Belmont: Principes éthiques et directives concernant la protection des sujets humains dans le cadre de la recherche, 1978 (DHEW Publication No. (OS) 78-0013 and No. (OS) 780014, for sale by the Superintendent of Documents, U.S. Government Printing Office, Washington, D.C. 20402.); Hubert Doucet, Au pays de la bioéthique. L'éthique biomédicale aux États-Unis, Genève, Labor \& Fides, 1996, aux pp. 44-47 [Doucet].

2. Instituts de recherche en santé du Canada, Conseil de recherches en sciences naturelles et en génie du Canada, Conseil de recherche en sciences humaines du Canada, Énoncé de politique des trois Conseils : Éthique de la recherche avec des êtres humains, août 1998 (avec les modifications de 2000, 2002 et 2005) [Énoncé de politique des trois Conseils]. 
formulaire de consentement doit alors faire l'objet d'un examen minutieux (trop minutieux?) ${ }^{3}$ par les CÉR compétents.

Or, depuis déjà quelques années, des chercheurs, des infirmières de recherche et des membres de CÉR expriment leur désarroi devant l'évolution des formulaires de consentement. On assiste à un accroissement de la quantité d'informations et à l'apparition de rubriques clairement porteuses de conséquences juridiques. On y retrouve en effet, de longues pages décrivant en détail toutes les interventions prévues auprès du sujet. On y lit aussi diverses clauses reliées aux aspects suivants : la nature et les objectifs du projet de recherche; son déroulement; la nature et la durée de la participation du sujet; le caractère volontaire de celle-ci; les critères d'exclusion; les bénéfices et les risques; les inconvénients de la participation; les traitements alternatifs; l'arrêt du projet par le chercheur ou le commanditaire; l'accès au dossier médical; la protection de la confidentialité des renseignements personnels; la conservation des données; l'autorisation de transmettre les résultats au médecin traitant; l'indemnité compensatoire pour les pertes et les contraintes subies; l'utilisation des résultats de recherche; leur possibilité de commercialisation; le financement du projet de recherche; les conflits d'intérêts du chercheur; les droits et les obligations ou responsabilités des sujets; l'indemnisation en cas de préjudice; l'identification des personnes ressources au sein du projet; la surveillance des aspects éthiques du projet; la formulation du consentement et la déclaration de la personne qui a recueilli le consentement écrit.

3. Certains auteurs reprochent même aux CÉR de consacrer trop de temps aux formulaires de consentement aux dépens d'une analyse plus large des projets : Michael McDonald, "Governance Relationships - The Governance of Health Research Involving Human Subjects (HRIHS)", Law Commission of Canada, Mai 2000, Section F : Conclusions and Recommendations, à la p. 5, en ligne: LCC <http://www.ethics.ubc.ca/people/mcdonald/ ccmacdonald.pdf> [Macdonald]; Charles Weijer, "The ethical analysis of risk" (2000) 28 J. L. Med. \& Ethics 344 [Weijer]. 
Les participants risquent fort d'être confondus par tant d'informations ${ }^{4}$, alors que les CÉR sont parfois surchargés par le travail qu'exige la révision de certains formulaires. On peut alors se questionner sur l'utilité de présenter aux sujets de recherche des formulaires aussi volumineux ${ }^{5}$. Contribuent-ils véritablement à une meilleure compréhension des enjeux du projet de recherche? Enfin, on peut aussi se demander si un document d'une telle ampleur éclaire réellement le consentement des sujets de recherche.

En droit, le formulaire de consentement constitue un élément du contrat intervenu entre le chercheur et le sujet et il porte quelques caractéristiques que l'on retrouve souvent dans les textes contractuels, tels que le détail de certains énoncés, la longueur imposante du document ou la signature des cocontractants. Il est inévitable que l'introduction d'un nombre considérable d'informations dans le formulaire de consentement donne à celui-ci un aspect que l'on trouve aussi dans les contrats de consommation ou certaines transactions commerciales. Or, la rationalité du droit contractuel n'est pas forcément compatible avec la protection des personnes. Une telle évolution des formulaires de consentement est-elle de nature à augmenter ou à diminuer la protection dont devraient bénéficier les sujets de recherche?

\section{A. LE FORMULAIRE DE CONSENTEMENT}

\section{La présence d'un écrit au contenu toujours plus détaillé}

De même que les membres des CÉR, nous constatons, de façon empirique, la tendance des commanditaires de la recherche à transformer les formulaires de consentement en de véritables

4. Angela Estey, Georgeann Wilkin et John Dossetor, "Are Research Subjects Able to Retain the Information They Are Given During the Consent Process" (1994) 3:2 Health Law Review 37 [Estey, Wilkin et Dossetor].

5. Cet aspect est ressorti notamment de plusieurs interventions lors d'un colloque organisé par le Réseau en santé respiratoire du FRSQ : "Éthique et intégrité scientifique en recherche médicale - Protection de la propriété intellectuelle», Montréal, 20 et 21 novembre 2003. 
contrats d'engagement réciproque. Par exemple, on retrouve dans ces formulaires des clauses d'exonération de responsabilité6, des affidavits précédant la signature, de multiples pages détaillant les moindres risques (ex. : douleur due à une piqûre), des obligations imposées aux sujets de recherche (divulgation, exécution, reconnaissance de la compréhension adéquate du formulaire, bonne foi), etc. Nous sommes mis en présence de documents de plus en plus exhaustifs. On semble parfois oublier que le contenu de ces formulaires devrait d'abord viser le respect de l'autodétermination et de l'inviolabilité de la personne ${ }^{7}$ et être considéré comme la manifestation écrite des informations données à une personne afin d'éclairer sa décision de participer à une recherche et de se soumettre aux inconvénients et aux risques que comporte celle-ci. La signature du sujet est exigée pour formaliser sa décision et éviter un choix frivole. Bref, comme il a déjà été dit ${ }^{8}$, le consentement est requis d'abord pour protéger le sujet de recherche et non le chercheur ou son commanditaire.

Comment, alors, expliquer la tendance à transformer le formulaire de consentement en contrat d'engagement volumineux comportant toutes sortes de stipulations secondaires, eu égard à la protection du sujet?

6. De telles clauses sont souvent contraires à la règle prévue à l'article 1474 C.c.Q., Code civil du Québec, L.Q. 1991, c. 64, art. 1474 voir infra note 103.

7. Patrice Deslauriers, "La responsabilité médicale et hospitalière" dans École du Barreau du Québec, Responsabilité, Cowansville, Éditions Yvon Blais, 2004 ; "l'examen par un C.E.R. des projets de recherche visant des majeurs aptes contribue à la protection de la dignité, du bien-être et des droits de tous les sujets»; Fonds de la recherche en santé du Québec, Guide d'éthique de la recherche et d'intégrité scientifique; Standards en éthique de la recherche et en intégrité scientifique du F.R.S.Q., 2e éd., Montréal, 2003, à la p. 20; Michel T. Giroux, "Le dilemme entre la protection des personnes inaptes et la recherche sur leur maladie" dans Service de la formation permanente du Barreau du Québec, Responsabilités et mécanisme de protection (2004), Cowansville, Yvon Blais, 2004.

8. François Tôth, "L'obligation de renseignement du médecin en droit civil québécois" dans Responsabilité médicale et hospitalière, Aspects éthiques et juridiques, Toronto, The Canadian Institute, 29 octobre 1990 [Tôth]. 
Le formulaire de consentement

(2007) 37 R.D.U.S. à la recherche : incompatibilité entre

le droit des personnes et le droit des contrats

Plusieurs explications peuvent être avancées. Tout d'abord, les règles éthiques et juridiques exigent la divulgation d'une quantité importante d'informations. D'une part, les tribunaux ${ }^{9}$ ont, avec le temps, rehaussé progressivement les exigences du devoir d'information des médecins et d'autre part, en 1996-1997, des règles éthiques ${ }^{10}$ et juridiques ${ }^{11}$ sont venues imposer la divulgation de toutes sortes d'informations dans le cadre de la recherche avec des sujets humains. La somme de renseignements à divulguer imposée par les organismes subventionnaires et les organismes de régulation vient naturellement grossir et complexifier les formulaires de consentement. Toutefois, cela n'explique pas tout. En effet, les compagnies commanditaires ont poussé cette logique à l'extrême en rédigeant des formulaires à rallonge (15 à 20 pages et même davantage) lesquels sont parfois réduits (10 ou 11 pages) après maintes négociations avec le CÉR.

9. Voir notamment : Haluska $v$. University of Saskatchewan, (1965) 53 D.L.R. (2d) 436 (Sask. C.A.); Hopp c. Lepp, [1980] 2 R.C.S. 192; Reibl c. Hughes, [1980] 2 R.C.S. 880, Weiss c. Solomon, [1989] R.J.Q. 731 (C.S. Qué.) [Weiss c. Solomon]; Pelletier c. Roberge, [1991] R.R.A. 726 (C.A. Qué.); Parenteau c. Drolet, [1994] R.J.Q. 689 (C.A.); voir aussi : Gerald Robertson, "Informed Consent Ten Years Later : The Impact of Reibl v. Hughes" (1991) 70 : 3 R. du B. Can. 423; Robert P. Kouri, "L'obligation de renseignement en matière de responsabilité médicale et la "subjectivité rationnelle": mariage de convenance ou mésalliance?" (1994) 24 R.D.U.S. 345; Pour un historique de la jurisprudence québécoise, voir Robert P. Kouri et Suzanne PhilipsNootens, L'intégrité de la personne et le consentement aux soins, 2e éd., Cowansville, Yvon Blais, 2005 aux pp. 313-316 [Kouri et Nootens].

10. Énoncé de politique des trois Conseils, supra note 2. Ces règles, du reste, ont presque un statut de règles juridiques: Angela Campbell et Kathleen Cranley Glass, "The Legal Status of Clinical and Ethics Policies, Codes, and Guidelines in Medical Practice and Research" (2001) 46 R.D. McGill 473.

11. Santé Canada, Les Bonnes pratiques cliniques: Directives consolidées Directive tripartite harmonisée de la Conférence internationale sur l'harmonisation des exigences techniques relatives à l'homologation des produits pharmaceutiques à usage humain, Ottawa, Ministre, Travaux publics et services gouvernementaux Canada, 1997, [Les Bonnes pratiques cliniques]; Règlement modifiant le règlement sur les aliments et drogues D.O.R.S./2001-203, art. C.05.005 (xi) (A) et C.05.010. Le caractère juridique des Bonnes Pratiques Cliniques naît selon nous de l'article C.05.010, de leur mention dans le résumé qui suit le règlement et de leur adoption par la Direction des produits thérapeutiques de Santé Canada. 
Le formulaire de consentement

à la recherche : incompatibilité entre (2007) 37 R.D.U.S. le droit des personnes et le droit des contrats

L'évolution des formulaires pourrait s'expliquer d'une deuxième façon. Puisque beaucoup de projets sont mis sur pied par des promoteurs situés aux États-Unis, on doit envisager l'hypothèse que cette tendance à transformer le formulaire de consentement en un contrat sophistiqué provienne du contexte juridique propre à notre voisin du Sud. En effet, confrontées à un excès de recours judiciaires dans le secteur de la santé, les compagnies pharmaceutiques américaines ont pris l'habitude d'inclure un nombre important de clauses visant, d'une manière défensive, à les protéger contre des poursuites ou des condamnations ${ }^{12}$. La traduction en français de ces formulaires n'a habituellement pas pour résultat de les simplifier, ni de les raccourcir.

Une troisième explication quant à la transformation des formulaires tient, selon nous, à la forme du consentement. Le fait même qu'un écrit soit légalement et éthiquement exigé13 afin de concrétiser le consentement nous paraît en grande partie responsable d'une certaine confusion et, du même coup, de l'exagération scripturaire. En effet, dans les textes normatifs applicables au Québec, l'écrit est généralement ${ }^{14}$ exigé lorsqu'une personne consent à se soumettre à une recherche ou à une expérimentation. Pourquoi cet écrit génère-t-il une certaine

12. J.-F. Burgelin, "La judiciarisation de la médecine" (2003) 3 Le Courier de l'éthique médicale 28 à la p. 33; "A signed consent form gives some legal protection to the hospital and its physicians, nurses and other health care providers should they later be sued by the patient for assault and battery or negligence where the patient alleges lack of informed consent»; William R. Hearn, "Consent to Medical Treatment: The Use of Consent Forms" (1992) 12 :3 Health Law in Canada 67 à la p. 67; D.R. Beyer, M.S. Lauer et S. Davis, "Readability of Informed Consent Forms [Letter]" (2003) 348 New Eng. J. Med. 2262.

13. Art. 24 C.c.Q.; Les Bonnes pratiques cliniques, supra note 11 art. 1.28; Énoncé de politique des trois Conseils, supra note 2 art. 2.1 b); Association médicale mondiale, Déclaration d'Helsinki, Washington, Assemblée générale de l'AMM, 2002, art. 22 [Déclaration d'Helsinki].

14. La Déclaration d'Helsinki, contrairement au Code civil du Québec, mais à l'instar de l'Énoncé de politique des trois Conseils n'en fait pas une exigence systématique : "Après s'être assuré de la bonne compréhension par le sujet de l'information donnée, le médecin doit obtenir un consentement libre et éclairé, de préférence par écrit", Déclaration d'Helsinki, ibid. 
Le formulaire de consentement

(2007) 37 R.D.U.S. à la recherche : incompatibilité entre

le droit des personnes et le droit des contrats

confusion? Parce que, comme nous tâcherons de le montrer plus bas, il est fondé sur deux rationalités juridiques distinctes qui ne sont pas toujours réconciliables.

\section{Pourquoi l'écrit est-il source de confusion?}

Notons, en préliminaire, qu'il est difficile de dire si la nature juridique de l'écrit pousse les rédacteurs à entrer dans une logique contractuelle extensive ou si, à l'inverse, la somme accrue d'informations contenue dans l'écrit engendre une confusion quant à sa nature ${ }^{15}$. Nous ne pouvons que supposer un lien entre les deux phénomènes, car si l'écrit n'existait pas, d'une part, l'acte d'informer (comme dans le cas des traitements médicaux) pourrait plus difficilement être confondu avec l'entente contractuelle entre le chercheur et le sujet de recherche et, d'autre part, la quantité d'informations donnée au sujet, serait forcément plus limitée à sa capacité d'écoute et aux limites de sa compréhension immédiate ${ }^{16}$. Bref, qu'elle soit la cause ou l'effet de la dispense d'une quantité accrue d'informations, l'incertitude quant à la fonction de l'écrit n'en demeure pas moins présente et traduit, selon nous, la poursuite de deux objectifs distincts.

\section{a) La protection du sujet de recherche}

Dans la logique de la bioéthique, la vocation première du consentement écrit à la recherche est, non pas la création d'obligations réciproques, mais la protection du sujet. La protection du sujet se trouve au cœur même des aspects

15. Il pourrait arriver que l'on considère l'écrit comme la totalité du processus de consentement, on risquerait alors d'en exagérer la portée et d'y consacrer toute l'attention, voir à ce sujet les remarques de McDonald et Weijer, supra note 3.

16. Estey, Wilkin et Dossetor, supra note 4; Wayne McKerrow, "Improving Patient Care and Reducing Risk Through Effective Communication" (1997) 18:1 Health Law in Canada 30; Laura B. Dunn et Dilip V. Jeste, "Enhancing Informed Consent for Research and Treatment" (2001) 24 Neuropsychopharmacology 595; Hubert O. Ballard et al., "Neonatal Research and the Validity of Informed Consent Obtained in the Perinatal Period", (2004) 24 Journal of Perinatology 409. 
Le formulaire de consentement à la recherche : incompatibilité entre (2007) 37 R.D.U.S. le droit des personnes et le droit des contrats

contractuels de la relation entre le chercheur et le sujet de recherche. C'est donc une notion centrale sur laquelle on ne saurait trop insister.

Le Code de Nuremberg (1947)17 ne prévoyait pas que le consentement soit exprimé par écrit. En 1979, le Rapport Belmont $^{18}$, qui a établi les bases de la bioéthique nordaméricaine ${ }^{19}$, n'en faisait pas non plus mention malgré le fait qu'il apparaisse déjà dans la première version de la Déclaration d'Helsinki (1964)20, et dans les versions qui ont suivi ${ }^{21}$. Au Canada, depuis 1987, la recherche subventionnée était encadrée 22 par les Lignes directrices concernant la recherche sur des sujets humains du Conseil de recherches médicales du Canada. Or, il y est clairement précisé que le formulaire de consentement ne devrait pas être conçu comme un contrat: "[l]e consentement donné par le sujet d'une expérience découle d'un rapport de

17. The Nuremberg Code, from trials of War Criminals before the Nuremberg Military Tribunals under Control Council Law No. 10. Nuremberg, October 1946 - April 1949. Washington D.C., U.S. G.P.O., 1949-1953.

18. Warrent T. Reich, dir., Encyclopedia of Bioethics, Vol. 5, New-York, Simon \& Schustser Macmillan, 1995. Voir aussi la traduction dans: Médecine et expérimentation, Cahiers de Bioéthique 4, Québec, P.U.L., 1982.

19. Doucet, supra note 1 à la p. 28.

20. «3c. Le consentement doit, dans la règle générale, être donné par écrit. La responsabilité d'une expérience sur un être humain incombe toujours à l'homme de science, elle n'incombe jamais au sujet qui se soumet de plein gré à l'expérience", 18e Assemblée générale de l'Association Médicale Mondiale, Helsinki, juin 1964; on peut retrouver ce texte dans: Henri Anrys, Les professions médicales et paramédicales dans le Marché Commun, Bruxelles, Maison Ferdinand Larcier, 1971, Annexe III.

21. Ex. : «9. Le médecin devra obtenir le consentement libre et éclairé du sujet, de préférence par écrit", 29e Assemblée générale de l'Association Médicale Mondiale, Tokyo, Octobre 1975. On peut trouver ce texte dans Gilbert Hottois et Marie-Hélène Parizeau, dir., Les mots de la bioéthique - un vocabulaire encyclopédique, Bruxelles, De Boeck-Wesmael, 1993 à la p. 113.

22. "[...] toute recherche financée par le Conseil doit se conformer à ces lignes directrices", Conseil de recherches médicales du Canada, Lignes directrices concernant la recherche sur des sujets humains, Ottawa, Ministre des Approvisionnements et Services Canada, novembre 1987 à la p. iii. 
Le formulaire de consentement

(2007) 37 R.D.U.S. à la recherche : incompatibilité entre

le droit des personnes et le droit des contrats

collaboration entre le chercheur et le sujet; il ne s'agit aucunement d'une obligation de type contractuel»23.

Selon cette approche, l'apposition d'une signature sur un formulaire de consentement ne devrait pas être perçue comme un engagement contractuel, car elle constitue simplement la manifestation de la volonté de collaboration qu'exprime un sujet de recherche. Pour ce dernier, ce geste de la signature constitue une étape supplémentaire dans l'expression de son choix comme pour "tempérer un excès d'enthousiasme passager»et l'obliger "à réfléchir avant de participer à un acte médical potentiellement risqué et ne comportant aucun avantage concret pour lui» 24 .

Malgré une obligation juridique de divulguer beaucoup d'informations ${ }^{25}$, l'écrit est donc avant tout exigé pour assurer davantage de sérieux dans l'obtention du consentement et la divulgation des risques et, de ce fait, pour protéger le signataire ${ }^{26}$. Le consentement écrit s'intègre dans ce que François Tôth préfère nommer "l'obligation de renseigner»27, plutôt que "l'obtention du consentement".

23. Ibid., à la p. 25.

24. Nous reprenons à notre compte ces passages formulés par Robert P. Kouri et Suzanne Philips-Nootens, "L'utilisation des parties du corps humain pour fins de recherche : l'article 22 du Code civil du Québec" (1994-95) 25 R.D.U.S. 359-396 à la p. 372 et ce, bien que ces auteurs ne nient pas, cependant, le fait que le formulaire de consentement tel qu'il est rédigé actuellement représente un véritable contrat.

25. Supra note 9.

26. "Le degré d'information à divulguer est donc déterminé en partie par le droit du patient d'être en mesure d'apprécier exactement ce à quoi il s'expose. Cet aspect est crucial dans la détermination de l'étendue de l'obligation d'informer et son standard d'appréciation" : Tôth, supra note 8 à la p. 9.

27. "Cette notion de consentement éclairé, traduction de l'expression américaine "informed consent", porte malheureusement à confusion. En effet, on peut comprendre que le patient n'est éclairé que s'il consent et que l'information doit viser le consentement du patient. Or cela est inexact. Le consentement du patient au traitement ou à l'opération n'est qu'une alternative possible de l'expression de la volonté éclairée du patient. Le patient peut refuser le traitement et ce refus libre et éclairé, est valide en 
Le formulaire de consentement à la recherche : incompatibilité entre (2007) 37 R.D.U.S. le droit des personnes et le droit des contrats

D’ailleurs, en droit civil, la nécessité du consentement écrit s'est toujours inscrite dans le cadre de l'inviolabilité de la personne 28 . C'est ainsi que le prévoyait l'Office de révision du code civil dans un rapport dont s'est inspiré le législateur québécois 29 en décembre 1971 lors de la première édition des dispositions relatives au corps humain (20 C.c.B.-C.) ${ }^{30}$ : "[e]n définitive, ce projet, comportant cinq articles, a pour objet de préciser la portée

droit. Aussi, est-il plus exact de parler d'obligation de renseigner», ibid., à la p. 8; Déjà en 1978, Bartha Maria Knoppers, dans Bartha Maria Knoppers, "Les notions d'autorisation et de consentement dans le contrat médical" (1978) 19 C. de D. 893 à la p. 899 réclamait une distinction entre le consentement et l'autorisation : "le consentement général crée la relation médecin-patient et assure un certain contrôle médical alors que l'autorisation porte plus spécifiquement sur une éventuelle intervention chirurgicale ou sur l'administration d'une anesthésie" [Knoppers].

28. Kouri et Nootens, supra note 9 à la p. 69; François Héleine, "Le dogme de l'intangibilité du corps humain et ses atteintes normalisées dans le droit des obligations du Québec contemporain" (1976) 36 R. du B. 2.

29. Voir à ce sujet les débats de l'Assemblée nationale concernant le Projet de loi no 89, Québec, Assemblée nationale, journal des Débats, 96, 30 novembre 1971 , aux pp. 4545-4558.

30. L'article proposé par l'Office de révision du Code civil (Rapport sur la reconnaissance de certains droits concernant le corps humain, Vol. 14, Montréal, 1971) se lisait comme suit: "Article 1: Le majeur peut, à titre gratuit et pour des fins thérapeutiques ou scientifiques, aliéner entre vifs une partie de son corps ou se soumettre à une expérience, pourvu que le risque couru ne soit pas hors de proportion avec le bienfait qu'on peut en espérer. Son consentement doit être donné par écrit et peut être révoqué». Avant 1971, rien n'était prévu dans le Code civil concernant l'expérimentation et la libre disposition de son corps. Depuis 1866, l'article 20 était rédigé, à un amendement près, de la façon suivante : "[e]st sujet britannique par droit de naissance, tout individu qui naît dans une partie quelconque de l'empire britannique, même d'un père étranger, et aussi celui dont le père est sujet britannique, quoique né lui-même en pays étranger; sauf dispositions exceptionnelles résultant des lois particulières de l'empire" [Rapport sur la reconnaissance de certains droits]. Voir PaulAndré Crépeau et John E.C. Brierley, Code civil 1866-1980, Montréal, SOQUIJ, 1981 à la p. 18; Voir aussi : W.F. Bowker, "Experimentation on Humans and Gift Tissue : arts. 20-23 of the Civil Code" (1973) 19 McGill L.J. 161; Albert Mayrand, L'inviolabilité de la personne humaine, Montréal, Wilson \& Lafleur, 1975 à la p. 1 [Mayrand]; Kouri et Nootens,, supra note 9 aux pp. 437 et 438. 
Le formulaire de consentement

(2007) 37 R.D.U.S. à la recherche : incompatibilité entre

le droit des personnes et le droit des contrats

du principe d'inviolabilité du corps humain dans le cadre du Code civil du Québec»31.

Plus de vingt ans plus tard, en 1993, ce même cadre interprétatif était confirmé dans les commentaires du ministre de la Justice suite à l'adoption de l'article 24 C.c.Q. en remplacement de l'article 20 C.c.B.-C : "[l]e nouvel article conserve l'exigence de l'écrit lorsqu'il s'agit de donner le consentement, mais il permet en tous les cas la révocation verbale, afin de mieux respecter la volonté de la personne ainsi que son droit à l'inviolabilité et à l'intégrité» 32 .

L'inviolabilité protège l'être humain, certes, mais pas de façon absolue ${ }^{33}$ et l'on peut porter atteinte à la personne d'autrui, lorsque cette personne y consent ou que la loi le permet $^{34}$. Le

31. Office de révision du Code civil, ibid.; Mayrand, ibid., aux pp. 11-16; "Consent is not only intented to protect the patient, his health and bodily integrity, but even more to protect the integrity of his humanity" : Samuel I. Shuman, "Informed Consent And Biomedical Research: A Historical Note" (1998) 18: 3\&4 L. Med. Q. 13.

32. Québec, Ministère de la Justice, Commentaires du ministre de la Justice, t. 1, Québec, Publications du Québec, 1993 à la p. 24. "[...] en ce cas, il va de soi que la motivation qui sous-tend cette exigence dépasse les intérêts particuliers des parties en cause; en effet, par l'imposition de l'écrit, le législateur ne vise pas seulement à s'assurer de la volonté d'un donneur particulier, mais tend aussi au respect du principe général de l'inviolabilité de la personne humaine", Didier Lluelles et Benoît Moore, Droit des obligations, Montréal, Thémis, 2006, à la note 109 aux pp. 574-575.

33. "Le principe de l'inviolabilité de la personne humaine, s'il était absolu, serait un peu gênant. L'homme ne tient pas à être intouchable en tout lieu, en tout temps et en toute circonstance. L'exclamation du Christ devant Marie-Magdelaine, Noli me tangere (Jean, 20, v. 17), convient à un Dieu qui s'est fait homme, non pas à un homme qui ne prétend pas être Dieu", Mayrand, supra note 30 à la p. 12 .

34. Mayrand, ibid.; voir notamment certaines lois à caractère public visant la protection de l'ensemble au détriment de certains droits individuels : Loi sur la santé publique, L.R.Q. c. S-2.2; Loi sur la protection des personnes dont l'état mental présente un danger pour elles-mêmes ou pour autrui, L.R.Q. c. P-38.001; Code criminel, L.R.C. 1985, c. C-46, ex. : article 45 (opérations chirurgicales), article 265 (voies de fait), article 257 al. 2 (immunité d'un médecin pour un prélèvement de sang) et 487.04 et ss. (analyse génétique effectuée à des fins médico-légales). 
formulaire de consentement ne fait donc que contribuer à l'encadrement permettant à l'individu d'autoriser une atteinte à sa personne.

Pourtant, malgré cet objectif réaffirmé de protection du sujet de recherche, un flou persiste quant à la fonction de cet écrit; un flou qui contribue peut-être à une incompréhension partagée entre les commanditaires de la recherche et les CÉR à l'égard du contenu des formulaires de consentement ${ }^{35}$. Nous expliquons cette confusion par l'existence d'une autre fonction attribuée à la forme écrite, celle de répondre de façon maximale aux exigences de la preuve en droit civil.

\section{b) La preuve écrite du consentement}

En droit, l'écrit constitue le meilleur moyen de preuve pour démontrer l'existence d'un acte juridique ${ }^{36}$. Chez les juristes praticiens, l'écrit est rédigé dans le but d'éviter un conflit et éventuellement, de servir de preuve lors d'un recours judiciaire. Il $\mathrm{y}$ aurait donc un intérêt à profiter du formulaire en y intégrant toutes les informations possibles afin d'offrir aux contractants une protection contractuelle qui soit la plus entière possible. Cette inclination à emplir le formulaire de consentement de toutes sortes de clauses semble, comme nous le verrons, avoir été entretenue depuis longtemps par l'objectif d'aménager une preuve.

Ainsi, l'Énoncé de politique des trois Conseils précise que "[d]ans notre société, une déclaration signée constitue généralement une preuve normale de consentement»37. L'écrit est perçu comme une preuve de consentement ${ }^{38}$. Avant l'Énoncé de

35. Les chercheurs, eux, se trouvent souvent coincés entre l'arbre et l'écorce.

36. L'article 2861 C.c. . donne la préférence à l'écrit pour faire la preuve d'un acte juridique : "Lorsqu'il n'a pas été possible à une partie, pour une raison valable, de se ménager la preuve écrite d'un acte juridique, la preuve de cet acte peut être faite par tous moyens"; voir aussi Pierre Tessier, "Les qualités et les moyens de preuve" dans École du Barreau du Québec, Preuve et procédure, Cowansville, Yvon Blais, 1995 à la p. 141.

37. Énoncé de politique des trois Conseils, supra note 2 à la p. 2.2.

38. Champoux Dumont c. Frenette, 2004 IIJCan 31742 (C.Q.). 
Le formulaire de consentement

(2007) 37 R.D.U.S. à la recherche : incompatibilité entre

le droit des personnes et le droit des contrats

politique des trois Conseils, les lignes directrices du Conseil de recherches médicales favorisaient aussi un consentement écrit permettant ainsi "la preuve du consentement»39. Elles rappelaient aussi qu'il s'agissait d'une "obligation légale de la province de Québec (voir l'article 20 du Code civil)»40. Qui plus est, lors de la rédaction de l'article 20 C.c.B.-C., l'Office de révision du Code civil situait le consentement écrit dans un cadre contractuel et expliquait son choix de la forme écrite par la facilité que celle-ci apporte en matière de preuve:

[...] cet article doit s'appliquer dans le cadre de la théorie générale des contrats et, par conséquent, la finalité de l'aliénation ou de l'expérience ne doit pas être contraire à l'ordre public. [...] Le souci de protéger plus efficacement encore le consentement du majeur et, le cas échéant, d'en faciliter la preuve, justifie le deuxième alinéa. Enfin, suivant la théorie générale des contrats, le majeur doit être sain d'esprit, libre et capable de comprendre les conséquences de son geste; de plus, le médecin ou l'expérimentateur a le devoir d'informer adéquatement le majeur des conséquences et des dangers que peut entraîner le don ou l'expérience ${ }^{41}$.

39. Lignes directrices concernant la recherche sur des sujets humains, supra note 22 à la p. 24; Pauline Lesage-Jarjoura et Suzanne Philips-Nootens, Éléments de responsabilité civile médicale, 2e éd., Cowansville, Yvon Blais, 2001 à la p. 167 [Lesaje-Jarjoura et Philips-Nootens]; Lorne Elkin Rozovsky, The canadian law of consent to treatment, $3 \mathrm{e}$ éd., Toronto, Butterworth, 2003.

40. Ibid.

41. Rapport sur la reconnaissance de certains droits, supra note 30 aux p. 11 et 12 ; Il est étonnant que l'Office emprunte la notion d'ordre public au droit contractuel alors que le droit des personnes en est lui-même imprégné : Jean Pineau et Serge Gaudet, Théorie des obligations, 4e éd., Montréal, Les Éditions Thémis, 2001 à la p. 313 [Pineau et Gaudet]. Cela montre à quel point, en 1971, cette législation québécoise touchant les droits de la personne était innovatrice. Actuellement, compte tenu des acquis et de notre expérience des droits de la personne, il ne nous viendrait sans doute pas à l'idée de passer par le droit contractuel pour appliquer l'ordre public à l'expérimentation. 
Dès son adoption, le nouvel article 20 C.c.B.-C. situait le consentement écrit dans un cadre contractuel. Toutefois, un cadre contractuel ne fait pas pour autant automatiquement du formulaire de consentement un contrat à part entière. Cette distinction peut se traduire de deux façons : d'une part, il pourrait y avoir un contrat signé sans qu'existe un consentement aux interventions, car il n'y aurait pas eu une information adéquate (ex. : sur les risques) et, d'autre part, un consentement écrit peut exister sans intégrer toutes les composantes du contrat. Dans ce deuxième cas de figure, l'écrit ne représenterait que la partie "consentement à l'expérimentation" (22 C.c.Q.) du contrat, le reste de l'entente étant verbal (1385 C.c.Q.).

Dans son traité sur la responsabilité, Jean-Louis Baudouin exprime fort bien la distinction entre la part contractuelle et la part inviolabilité de la personne que comporte l'obligation d'informer dans la relation médecin/patient :

[1]e fondement juridique de l'obligation d'informer peut, à un premier niveau, être recherché à partir du postulat conférant une base contractuelle à la relation professionnelle: l'existence et la validité même du contrat médical exigeraient un consentement présent, libre et éclairé. Il peut aussi (et surtout à notre avis) être désormais relié directement au principe plus général et plus fondamental de l'inviolabilité de la personne humaine, principe reconnu et consacré tant par la Charte des droits et libertés de la personne que par le Code civil. La personne humaine étant inviolable, on ne saurait y porter atteinte sans, au préalable, avoir obtenu d'elle un consentement libre et éclairé à l'acte. Les articles 10 et 11 du Code civil codifient clairement cette règle en étendant désormais le devoir d'information à toute espèce d'intervention (traitement, tests, diagnostic, examens, expérimentation, etc.) ${ }^{42}$.

42. Jean-Louis Baudouin et Patrice Deslauriers, La responsabilité civile, Cowansville, Yvon Blais, 2003 au nº 1466 aux pp. 1011-1012. 
Le formulaire de consentement

(2007) 37 R.D.U.S. à la recherche : incompatibilité entre

le droit des personnes et le droit des contrats

Jean-Louis Baudouin reconnaît donc bien deux approches face au consentement: l'approche contractuelle, en vertu de laquelle un contrat médical est formé lorsqu'il $y$ a eu un consentement et l'approche du droit à l'inviolabilité personnelle. Celui qui interprète la nécessité du consentement dans le seul contexte contractuel dénature ce dont il s'agit, car il ne tient pas compte du droit à l'inviolabilité personnelle. Pourtant, dans leur traité, désormais classique, sur les obligations, Jean-Louis Baudouin et Pierre-Gabriel Jobin présentent l'obligation de renseigner du médecin comme un élément essentiel à la formation du contrat :

i. Obligation d'information à la formation du contrat

- Obligation générale d'information

319 - L'obligation d'une partie de fournir à l'autre les informations nécessaires à un consentement pleinement éclairé est reconnue depuis assez longtemps dans certains domaines. C'est le cas notamment du contrat de soins médicaux. Eu égard aux conséquences possibles sur l'intégrité corporelle, valeur reconnue et protégée par la Charte et le Code civil, la jurisprudence a développé certaines règles concernant la divulgation, par les médecins à leurs patients, des risques probables et parfois même possibles des soins ou interventions recommandées ${ }^{43}$.

Le fait que ces auteurs placent le consentement aux soins sous la rubrique de la formation du contrat pourrait engendrer une certaine confusion sur l'objet du consentement. Nous dirions qu'il faut en faire une lecture qui dépasse le seul aspect de l'obligation d'information à la formation du contrat et qui embrasse l'inviolabilité personnelle à titre de valeur sanctionnée par les droits de la personne.

43. Jean-Louis Baudouin et Pierre-Gabriel Jobin avec la collaboration de Nathalie Vézina, Les Obligations, 6e éd., Cowansville, Yvon Blais, 2005 à la p. 353 [Baudouin, Jobin et Vézina]. 


\section{c) Le droit des personnes et le droit des contrats}

On comprend d'autant plus ce type de confusion ponctuelle lorsqu'on observe les similarités entre les dispositions légales relatives aux soins et aux contrats, lesquelles se côtoient fréquemment dans le droit des obligations ${ }^{44}$. Par exemple, la nature libre et éclairée du consentement concerne aussi bien le caractère licite de la fourniture de soins (art. 10 et 11 C.c.Q.) que les conditions de formation du contrat (art. 1399 C.c.Q.). De même, dans les deux cas, la capacité des personnes ou leur aptitude à consentir est un aspect important dont dépend la validité du consentement (art. 21 et 1398 C.c.Q.). Les deux domaines du droit ont aussi aménagé des modalités particulières dans le cas des mineurs et des personnes inaptes à consentir ${ }^{45}$. On constate donc la présence de dispositions similaires dans deux domaines du droit (le droit des personnes ${ }^{46}$ et le droit des contrats) pourtant différents.

Le contexte contractuel justifierait donc la tendance à produire un contrat en guise de formulaire de consentement, c'està-dire un document qui vise l'exhaustivité des modalités de l'entente et dans lequel tout le déroulement du projet est décrit dans le menu détail (ex. : le médecin de l'étude vous demandera de vous allonger avant la prise de sang); un document dans lequel on retrouve des limitations à l'indemnisation (ex. : le commanditaire ne paiera pas pour les pertes de salaire en cas d'accident lié à l'étude ou au médicament ${ }^{47}$ ); un document dans lequel la gestion

44. Ibid. à la p. 1 .

45. Voir par exemple, les articles 11-21, 157, 161, 283, 287, 290, 1385, 1398, 1405, 1409 C.c.Q. etc.,.

46. Bien que le concept de droit des personnes semble être maintenant accepté et entériné par la législation, la chose a suscité une certaine controverse; Roger Nerson, Les droits extrapatrimoniaux, Paris, L.G.D.J., 1939 aux pp. 347 et ss. [Nerson, "Droits extrapatrimoniaux"]; Pierre Kayser, "Les droits de la personnalité aspects théorique" (1971) 69 Rev. Dr. Civ. 445; Kouri et Nootens, supra note 9 à la p. 73.

47. Notez qu'en ce qui concerne ce type de clause, le juriste est mal outillé pour faire valoir l'interdit prévu à l'article 1474 C.c.Q, car il ne s'agit pas, à proprement parler, d'une exclusion de responsabilité. Certains CÉR cherchent néanmoins à limiter ce type de clause dont l'effet sur les sujets- 
Le formulaire de consentement

(2007) 37 R.D.U.S. à la recherche : incompatibilité entre

le droit des personnes et le droit des contrats

de l'information personnelle ne tient pas dans une page, car on reproduit les droits et obligations prévus dans la Loi sur la Protection des renseignements personnels et les documents électroniques ${ }^{48}$; un document dans lequel on prévient du risque de la douleur et du bleu causés par la piqûre d'une seringue; un document que signeront les sujets, leur témoin, l'infirmière de recherche (qui informe et fait signer) et le chercheur.

On reconnaît dans ces manifestations la logique contractuelle qui anime les rédacteurs. En se fondant sur le contexte contractuel et en l'absence de dispositions contraires dans la loi, il est possible qu'un tribunal entérine la légitimité de toutes sortes de stipulations. Par exemple, la décision de la Cour suprême dans l'affaire Frenette c. La Métropolitaine ${ }^{49}$, ne confirmait-elle pas la toute puissance de la signature pour donner un accès potentiellement illimité aux dossiers médicaux du signataire d'un contrat d'assurance vie ${ }^{50}$ ? Pourtant, même si elle considère le formulaire comme un contrat, la loi permet au sujet de se retirer de façon unilatérale en tout temps et sans aucune formalité. Ce droit légal est certainement exceptionnel en matière contractuelle où, d'une manière générale ${ }^{51}$, la parole donnée prend force de loi entre les parties. Il vient rappeler que l'objet du contrat de recherche n'est pas anodin. Même dans le cadre "formaliste» 52 $\mathrm{du}$ droit contractuel, le Code civil nous rappelle que

patients non juristes leur semble être le même qu'une véritable exclusion de responsabilité.

48. Loi sur la Protection des renseignements personnels et les documents électroniques, L.C. 2000 , c. 5.

49. Frenette c. La Métropolitaine, [1992] 1 R.C.S. 647.

50. Ainsi s'exprimait Mme la Juge L'Heureux-Dubé : "[...] le droit à la nondivulgation des dossiers médicaux appartient au patient et [...] celui-ci peut y renoncer inconditionnellement ou conformément aux conditions qu'il prescrit dans l'autorisation de divulguer", ibid. à la p. 672.

51. Les exceptions sont rares. Pensons à l'article 59 de la Loi de protection du consommateur, L.R.Q., c. P-40.1 qui permet la résiliation unilatérale par le consommateur dans les dix jours d'une vente effectuée par un vendeur itinérant.

52. Dans lequel l'accomplissement des formalités est étroitement lié aux droits et obligations : un écrit, des témoins, un objet, une cause, une offre et une acceptation, un avis écrit, etc. 
l'expérimentation réalisée sur des personnes doit respecter scrupuleusement les droits fondamentaux, dont le droit à l'intégrité personnelle qui permet au sujet d'exprimer la volonté de se retirer d'une recherche à laquelle il a d'abord consenti. Nous sommes donc en présence d'un écrit qui, pour son interprétation juridique, fait appel tant au droit des personnes ${ }^{53}$ qu'au droit des contrats. Or, ces deux champs d'interprétation ne sont pas forcément compatibles et ce, même si l'un fait parfois appel aux règles de l'autre ${ }^{54}$. Le fait que des personnes passent des contrats ne signifie pas forcément que le droit des contrats couvre tous les aspects des relations interpersonnelles. Le consentement écrit à l'expérimentation pourrait en être un bon exemple comme nous le verrons dans ce qui suit.

\section{B. LE DROIT DES PERSONNES ET LE DROIT DES CONTRATS SONT-ILS DES VASES COMMUNICANTS?}

En utilisant la métaphore des vases communicants, nous voulons exprimer ici la possibilité d'importer des notions de droit contractuel dans le droit des personnes tels deux vases que l'on pourrait transvider l'un dans l'autre sans dénaturer leur contenu.

La relation entre le chercheur et le sujet de recherche est une relation de nature contractuelle. À ce sujet, l'article 1385 C.c.Q se lit comme suit :

[1]e contrat se forme par le seul échange de consentement entre des personnes capables de contracter, à moins que

53. Le droit des personnes n'est pas une notion bien définie ni acceptée par tous. "Ces droits en effet, présentent des contours incertains. Il existe plusieurs écoles de pensée sur le sujet", Edith Deleury, "Une perspective nouvelle : le sujet reconnu comme objet du droit" (1972) 13 C. de D. 529 à la p. 530 [Deleury, "Perspective nouvelle»]. Nous n'employons ce concept que pour distinguer le droit contractuel d'un droit visant la protection de l'inviolabilité de la personne. Pour une remise en question des droits de la personnalité voir notamment Paul Roubier, Droits subjectifs et situations juridiques, Paris, Dalloz, 2005; ou Michel Villey, Le droit et les droits de l'homme, 3e éd., Paris, P.U.F., 1998 [Villey].

54. Voir par exemple l'article 1409 C.c.Q.: "Les règles relatives à la capacité de contracter sont principalement établies au livre Des personnes". 
Le formulaire de consentement

(2007) 37 R.D.U.S. à la recherche : incompatibilité entre

le droit des personnes et le droit des contrats

la loi n'exige, en outre, le respect d'une forme particulière comme condition nécessaire à sa formation, ou que les parties n'assujettissent la formation du contrat à une forme solennelle.

Il est aussi de son essence qu'il ait une cause et un objet [nous soulignons].

En l'occurrence, le chercheur consent à "utiliser" des sujets de recherche à des fins de collecte de données et, de leur côté, les sujets consentent à se prêter aux interventions. En toile de fond, les deux contractants espèrent contribuer à l'avancement de la connaissance "scientifique»55. Dans la recherche sur des sujets humains, la volonté de faire progresser la connaissance et d'améliorer les traitements peut être commune aux chercheurs et aux sujets. Donc, conformément à l'article 1385 C.c.Q., le contrat en matière d'expérimentation comporte un échange de consentement entre des personnes capables de contracter (directement ou par l'intermédiaire d'un tiers ${ }^{56}$ ), une cause l'avancement de la connaissance et/ou l'amélioration de la santé

55. Il n'est pas certain que la finalité de tous les projets de recherche soit de faire avancer la science. En effet, l'on voit parfois des projets visant à vérifier la qualité des actes d'une profession ou visant à tester un appareil médical. Dans ces cas, bien que la méthode soit parfaitement rigoureuse et fasse appel à des sujets humains, l'objectif répond d'abord à un besoin d'efficacité ou d'efficience sociale et non d'avancement du savoir scientifique. Dans ces situations la science nous semble être au service de la technique, ce qui n'est pas forcément, en soi, une mauvaise chose. Voir à ce sujet: Julien P. Cabanac, L'influence concurrente de la science et de la technique en droit de la santé, Essai de maîtrise en droit, Université de Sherbrooke, mai 1999 [non publié].

56. On ne réfère pas ici au consentement aux soins des articles 15 et 21 C.c.Q., mais au consentement libre et éclairé du contrat (art. 1399 C.c.Q.); consentement qui peut être donné par le représentant légal en stipulant pour le sujet de recherche. Dans le cas de l'expérimentation, il nous apparaît illusoire que le mineur de plus de 14 ans puisse passer une entente avec le chercheur alors que ce n'est pas lui qui consent à l'expérimentation (à ce sujet, voir : Lesage-Jarjoura et Philips-Nootens, supra note 39 à la p. 15). Cela ne signifie pas pour autant que dans le cas particulier de l'expérimentation sur des mineurs de plus de 14 ans, l'article 21 C.c.Q. soit l'assise juridique permettant l'entente contractuelle avec le chercheur. À cet égard, d'autres règles juridiques pourraient trouver une certaine application : la promesse pour autrui ou la tutelle au mineur. 
du sujet ou d'autres personnes) et un objet (le déroulement du protocole de recherche). Certaines dispositions qui concernent les contrats en général peuvent trouver application en matière d'expérimentation. C'est le cas, par exemple, de l'article 1403 C.c.Q. qui établit un vice de consentement dans la situation où une crainte est engendrée par "l'exercice abusif d'un droit ou d'une autorité ou par la menace d'un tel exercice». Dans le contexte de la recherche, nous pouvons penser à la possibilité qu'un chercheur/médecin fasse pression sur son patient en affirmant qu'un refus de participer à la recherche aurait pour conséquence de le priver des soins qu'il reçoit déjà ${ }^{57}$.

\section{L'indisponibilité de la personne et le commerce}

Grégoire Loiseau constate que le concept de "l'indisponibilité de la personne»58 est susceptible de s'estomper, laissant le corps humain disponible aux lois du commerce59. Selon G. Loiseau, le caractère intouchable de l'être humain à travers son corps s'exprimait jadis par des règles prohibitives. Or, au fur et à mesure des avancées de la science, le corps a perdu son caractère sacré, permettant aux individus d'en user selon leur volonté. Avec la disparition du caractère sacré du corps et les manipulations scientifiques des tissus humains, on se rapproche du droit commercial et du principe de l'autonomie de la volonté. Il ne reste ensuite qu'un pas à franchir entre le droit du commerce et la logique commerciale. Ce glissement du sacré vers le commercial conduit à penser que le droit commercial s'impose parce que la

57. Art. 1403 C.c.Q. : "La crainte inspirée par l'exercice abusif d'un droit ou d'une autorité ou par la menace d'un tel exercice vicie le consentement». De façon plus subtile, on pourrait fort bien concevoir la crainte de certains patients rendus vulnérables par la maladie devant l'insistance de leur médecin ou du personnel infirmier dont dépend leur qualité de vie et parfois même leur espérance de vie. Dans ces cas particuliers, le devoir d'information devrait être exécuté avec beaucoup de tact. Voir à ce sujet Mireille D. Castelli et Marlène Cadorette, "L'expérimentation biomédicale et l'inviolabilité de la personne : autodétermination ou protection de l'intégrité physique" (1994) 25 R.G.D. 173 aux pp. 204-205 [Castelli et Cadorette].

58. Nerson, "Droits extrapatrimoniaux", supra note 46 aux p. 394 et ss.

59. Grégoire Loiseau, "Le rôle de la volonté dans le régime de protection de la personne et de son corps" (1992) 37:4 R.D. McGill 965. 
Le formulaire de consentement

(2007) 37 R.D.U.S. à la recherche : incompatibilité entre

le droit des personnes et le droit des contrats

personne tombe de son piédestal. Or, ce n'est pas parce que le corps humain perd de son caractère sacré que les dispositions protectrices du corps humain sont remplaçables par les lois de la propriété et du commerce. Le droit des personnes et le droit des contrats ne sont pas deux vases communicants permettant d'appliquer la logique contractuelle à la personne comme si sa dignité lui avait été retirée. Le fait de rendre chaque personne responsable de son corps ne transporte pas automatiquement les décisions des individus vis-à-vis de leur personne dans un contexte exclusivement contractuel60. Rappelons que dans la recherche avec des êtres humains, l'objet du contrat est réalisé sur un être humain. Ainsi, même si la relation chercheur/sujet était de nature contractuelle, même si le consentement à l'expérimentation était vu comme une modalité du contrat entre le chercheur et le sujet, ce consentement, comme nous l'avons vu plus haut, fait aussi appel au droit des personnes.

L'abandon de certains interdits concernant le corps a pour effet de le rendre commercialisable, c'est-à-dire de le placer au même niveau que les biens sujets au commerce et de le soumettre partiellement au champ juridique du droit contractuel61. Ce n'est d'ailleurs pas de tout temps que le corps humain a été hors commerce et donc hors du droit du commerce ${ }^{62}$. Nous voulons exprimer ici la présence distincte de deux sources du droit dont les règles, même si elles se ressemblent, poursuivent des fins différentes et doivent donc être interprétées différemment, empêchant l'interprète de puiser, sans précaution 63 dans la jurisprudence, la doctrine ou même la logique de l'un pour appliquer l'autre.

60. Kouri et Nootens, supra note 9 à la p. 67.

61. Édith Deleury, "La personne en son corps : l'éclatement du sujet" (1991) 70 R. du B. Can. 448; Marie-Angèle Hermitte, "Le corps hors du commerce, hors du marché» (1988) 33 Arch. phil. Droit 323 [Hermitte].

62. Hermitte, ibid. à la p. 325 .

63. Édith Deleury met bien en lumière les écueils moraux et juridiques résultant potentiellement de l'application du droit des biens lorsque l'être humain devient objet de contrat, Deleury, "Perspective nouvelle", supra note 53. Voir aussi Nerson, "Droits extrapatrimoniaux", supra note 46 aux p. 355 et ss. 
Poussant plus loin le raisonnement, il conviendrait de se demander si, dans le contexte du consentement à l'expérimentation, le droit des personnes ne devrait pas jouir d'une prépondérance sur le droit des biens.

Dans le Code civil du Québec, le consentement à l'expérimentation est abordé à la section I (Des soins) du chapitre premier du titre deuxième du livre premier intitulé : Des personnes. Ainsi, la simple classification du Code nous induirait à penser que, en matière de consentement aux soins, le droit des personnes est le premier concerné. Toutefois, même s'il existe une certaine rationalité dans la construction des codes modernes $^{64}$, le juriste est accoutumé à passer d'un chapitre à l'autre, l'un servant à interpréter l'autre, le Code étant un tout ${ }^{65}$. On ne saurait donc invoquer la logique de l'organisation comme seul argument pour revendiquer la priorité du droit des personnes en matière de soins.

On ne saurait non plus proposer la nature "d'ordre public" inhérente au droit des personnes, car le droit contractuel est aussi assujetti à l'ordre public 66 et de nombreuses dispositions du droit des contrats sont d'ordre public, ce concept n'étant lui-même pas bien défini 67 . Malgré tout, le chapitre relatif aux soins est entièrement d'ordre public. Nous devons ici soulever la nuance entre un chapitre dont toutes les dispositions sont d'ordre public

64. Jean Ray, Essai sur la structure logique du Code civil français, Paris, Librairie Félix Alcan, 1926 aux pp. 193 et ss.

65. Ibid.

66. Art. 1413 C.c.Q.: «Est nul le contrat dont l'objet est prohibé par la loi ou contraire à l'ordre public". Lors de l'adoption de l'article 20 C.c.B.-C., les notes explicatives de l'Office de révision du Code civil expliquaient clairement que le respect de l'ordre public provenait du fait que cet article s'appliquait "dans le cadre de la théorie générale des contrats", Rapport sur la reconnaissance de certains droits, supra note 30.

67. "Cet article sanctionne de nullité le contrat dont l'objet est illégal, illicite ou immoral", ministre de la Justice, Commentaires du ministre de la Justice, supra note 32 à la p. 858, sous l'article 1413; "L'ordre public serait cet ordre flou et changeant, qui prime les intérêts privés et qui est nécessaire au maintien de l'organisation sociale ou au mieux-être de la société en général», Pineau et Gaudet, supra note 41 à la p. 311. 
Le formulaire de consentement

(2007) 37 R.D.U.S. à la recherche : incompatibilité entre

le droit des personnes et le droit des contrats

et un chapitre dont les dispositions doivent être appliquées dans le respect de l'ordre public 68 . En matière de soins, cette nuance milite fortement en faveur d'une priorité du droit des personnes sur le droit contractuel.

Enfin, le critère majeur de distinction entre les deux champs du droit réside dans celui décrit notamment par Dominique Thouvenin 69 et fort bien mis en application par Édith Deleury ${ }^{70}$ lorsqu'elle distingue le corps sujet et le corps objet, le premier fondant le droit des personnes, le second faisant appel au droit des biens.

L'inviolabilité de la personne protège celle-ci en tant que sujet de droit ${ }^{71}$. Il s'agit d'un droit hors commerce, extrapatrimonial, sans valeur marchande ${ }^{72}$. À l'inverse, "[l]es droits patrimoniaux constituent l'ensemble des droits de caractère économique et appréciables en argent [...]"73. Sans remonter aux sources nominalistes de la notion de sujet de droit ${ }^{74}$, rappelons

68. Dans le droit des personnes, on ne peut déroger aux dispositions du C.c.Q., car elles sont d'ordre public alors qu'en droit contractuel, sauf pour certaines dispositions, l'ordre public n'est qu'une limite à la liberté de contracter.

69. Dominique Thouvenin, "Quand le droit prend le corps", Actes, (1985) 49-50 Cahiers d'action juridique 35; Xavier Dijon, Le sujet de droit en son corps, Bruxelles, Larcier, 1982 aux pp. 672-675.

70. Deleury, "Perspective nouvelle», supra note 53.

71. Jean Carbonnier, Droit civil - 1/Les personnes, $21^{\mathrm{e}}$ éd., Paris, P.U.F., 2000 aux pp. 15 et ss. [Carbonnier]; Roger Nerson, "De la protection de la personnalité en droit privé français" dans Travaux de l'Association Henri Capitant pour la culture juridique française, T. XIII, Paris, Dalloz, 1963 à la p. 61 [Nerson, "Protection de la personnalité»].

72. Henri Capitant, Introduction à l'étude du droit civil, 4e éd., Paris, A. Pedone, 1925 aux pp. 135 et ss. [Capitant]; Édith Deleury et Dominique Goubau, Le droit des personnes physiques, 3e éd., Cowansville, Yvon Blais, 2002 à la p. 75 [Deleury et Goubeau]; Baudouin, Jobin et Vézina, supra note 43 à la p. 1; Deleury, "Perspective nouvelle», supra note 53 à la p. 532.

73. Baudouin, Jobin et Vézina, ibid. à la p. 2.

74. André-Jean Arnaud, Les origines doctrinales du Code civil français, Vol. IX, Paris, L.G.D.J., 1969; Ph-I. André-Vincent, Les révolutions et le droit, vol. XVIII, Paris, L.G.D.J., 1974; Villey, supra note 53; Michel Bastit, Naissance de la loi moderne, Paris, P.U.F., 1990. Michel Villey explique que Guillaume d'Occam a popularisé le nominalisme au XIV siècle. Tranchant avec la 
Le formulaire de consentement

à la recherche : incompatibilité entre (2007) 37 R.D.U.S.

le droit des personnes et le droit des contrats

que le droit des personnes reconnaît l'être humain à travers ses attributs comme une réalité distincte des choses ${ }^{75}$. Certains attributs de la personnalité sont tellement valorisés qu'ils ont été élevés au rang de droits fondamentaux dans diverses chartes, déclarations et constitutions ${ }^{76}$. Les droits de la personnalité constituent donc "une catégorie originale" 77 qui est rattachée à la personne, notamment dans sa dimension corporelle. Ce corps qui

vision grecque du Cosmos, Occam plaçait l'individu au centre de la relation à Dieu. La table était mise pour que le droit évolue dans ce nouveau contexte philosophique. Chacun pouvait devenir sujet de droit, recentrant ainsi l'ensemble du droit autour de l'individu. L'individu devenant désormais le centre des préoccupations, le droit ne dépendait plus de l'équilibre de l'univers, mais dépendait de la volonté des décideurs ou des interprètes et donc de leur rationalité. De là apparaissait le droit naturel moderne dont l'essence serait de générer des règles juridiques à partir d'une vision rationnelle du monde.

75. Capitant, supra note 72 à la p. 133; Carbonnier, supra note 71. Nous distinguons ici le droit des personnes du droit subjectif décrit par Jacques Ghestin et Gilles Goubeaux dans Traité de droit civil - Introduction générale, 3e éd., Paris, L.G.D.J., 1990 à la p. 144; le second englobant le premier. En effet, le droit subjectif est un droit fondé sur la notion de sujet de droit. Or, le contrat du Code civil naît d'un accord de volonté entre deux sujets. La notion juridique contemporaine de contrat fait donc appel, elle aussi, à un droit subjectif, c'est-à-dire un droit fondé sur l'individu, ex. : le créancier distinct de la créance : Pineau et Gaudet, supra note 41 aux p. 2 et 12; Nerson, "Protection de la personnalité", supra note 71.

76. Charte canadienne des droits et libertés, partie I de la Loi constitutionnelle de 1982, constituant l'annexe B de la Loi de 1982 sur le Canada (R.-U.), 1982, c. 11; Déclaration canadienne des droits, L.R.C. 1985, App. III; Charte des droits et libertés de la personne, L.R.Q. c. C-12; Déclaration universelle des droits de l'homme, Rés. AG 217 (III), Doc. Off. AGNU, 3e sess., supp. $\mathrm{n}^{\circ} 13$, Doc N.U. A/810 (1948) 71; The American bill of rights (1791); Déclaration des droits de l'homme et du citoyen de 1789. Ces documents sont reproduits en tout ou en partie dans André Morel, Code des droits et libertés, 6e éd., Montréal, Thémis, 1998. Certains diront que le terme "élevé" est mal choisi car ces droits ont commencé à exister dans un cadre publiciste, c'est-à-dire dans une relation des citoyens avec l'État. Les premiers droits de la personne étaient inscrits dans les premières grandes déclarations (ex. :1776 et 1789). Ces droits n'ont été importés dans le droit privé que par la suite. Voir à ce sujet : René Savatier, Les métamorphoses, 3e série, Paris, Dalloz, 1959 à la p. 7; Nerson, "Protection de la personnalité", supra note 71 à la p. 64 .

77. Deleury et Goubau, supra note 72 à la p. 74 . 
Le formulaire de consentement
(2007) 37 R.D.U.S. à la recherche : incompatibilité entre
le droit des personnes et le droit des contrats

"échappe au monde des objets»" ${ }^{78}$ se trouve "triplement défendu : $1^{\circ}$ contre les atteintes des tiers par une sorte d'interdit, l'inviolabilité; $2^{\circ}$ contre le pouvoir de disposition de l'individu de lui-même, par des restrictions à l'autonomie de la volonté; $3^{\circ}$ contre le regard d'autrui, une défense que l'on nomme pudeun 79 . En bref, selon une perspective historique, le droit des personnes est apparu en marge du droit patrimonial. D'un coup d'œil rapide, ce droit (des personnes) pourrait donc être circonscrit de manière négative par ce qui n'est pas du droit patrimonial, alors qu'il est de l'essence même des contrats de faire appel aux biens et à la notion de patrimoine. Vis-à-vis de notre réflexion sur le formulaire de consentement à la recherche, cette opposition mérite d'être examinée plus en détail.

Dans les prochaines pages, nous montrerons d'une part, que le consentement à l'expérimentation accorde une priorité au respect de l'être humain. D'autre part, nous exposerons quelques conséquences concrètes que risquerait d'entraîner une hégémonie du droit contractuel à l'égard du formulaire de consentement afin de mettre en lumière une incompatibilité entre cette logique du droit et la protection des sujets de recherche.

\section{Une liberté contractuelle limitée}

Le sujet de recherche est à la fois un contractant et la personne sur laquelle est réalisé l'objet du contrat de recherche. Puisque cette personne est dotée de la dignité humaine et de droits fondamentaux, la relation contractuelle entre le chercheur et le sujet de recherche doit tenir compte en priorité de la dignité humaine et de la protection personnelle du sujet.

En ce qui concerne la formation du consentement, ce constat implique que le sujet doive être éclairé convenablement (art. 11 C.c.Q.). De ce point de vue, la longueur du formulaire n'est pas nécessairement importante en elle-même; il pourrait même être relativement long à la condition de respecter des limites

78. Carbonnier, supra note 71 à la p. 20.

79. Ibid. 
raisonnables. L'important est que le chercheur s'assure que le sujet ait bien intégré l'information contenue dans le formulaire. On pourrait même avancer que si l'information intégrée par les sujets était à l'image du formulaire de consentement, les CÉR encourageraient de volumineux formulaires. Malheureusement, la réalité pratique nous montre que les formulaires ne sont pas toujours bien expliqués et que, même lorsque le chercheur prend le temps d'expliquer, la somme d'informations est telle que la plupart des sujets n'en retiennent qu'une partie 80 .

Il est important d'insister sur ce point, car l'ampleur des formulaires ne constitue un problème que dans la mesure où l'authenticité du consentement s'en trouve affecté. Le comportement $\mathrm{du}$ chercheur ou du personnel de recherche constitue donc un facteur primordial dans l'évaluation de ce que l'on considère être une quantité acceptable d'informations. Toutefois, il faut savoir que les sujets ne peuvent pas assimiler trop d'informations d'un seul coup et ce, malgré tous les efforts louables $\mathrm{du}$ chercheur ${ }^{81}$. C'est la raison pour laquelle un document du Comité de liaison en éthique de la recherche de l'Université de Montréal (CLÉRUM) propose de limiter la longueur des formulaires à 5 ou 6 pages $^{82}$.

La nature même du consentement à l'expérimentation le distingue catégoriquement du consentement à un engagement purement contractuel. En effet, le sujet de recherche dispose de la capacité de se retirer de la recherche, tout au long de son déroulement. Le consentement à l'expérimentation existe à titre de continuum et, à ce titre, il n'est absolument pas limité au moment requis pour son expression écrite :

[1]e moment où le sujet éventuel signe le formulaire de consentement et devient sujet de recherche ne détermine

80. Supra note 16.

81. Estey, Wilkin et Dossetor, supra note 4.

82. Sonia Audy, Rédiger un formulaire de consentement respectueux de l'autonomie des sujets pressentis: une mission impossible?, Montréal, CLÉRUM, 14 mars 2005 à la p. 22 [Audy]. 
Le formulaire de consentement

(2007) 37 R.D.U.S. à la recherche : incompatibilité entre

pas la volonté qui prévaudra lors des moments ultérieurs, puisque le sujet peut se retirer de l'expérimentation à tout moment avant que celle-ci ne prenne fin. En conséquence, le consentement ne doit pas être perçu comme un événement fini et limité à un moment précis, mais comme l'expression d'une volonté continue dans le temps.

L'idée de continuum représente la permanence, l'écoulement ininterrompu, le caractère incessant de quelque chose qui est situé dans l'espace ou dans le temps. Cette idée se trouve opposée à celles d'interruption, de rupture et de discontinuité. En ne révoquant pas le consentement qu'il a exprimé, le sujet réaffirme à tout moment sa volonté de participer à

l'expérimentation. Conceptuellement, l'absence de révocation $\mathrm{du}$ consentement équivaut à répéter l'expression implicite du consentement ou à renouveler celui-ci de manière à ce qu'il se perpétue. Cette expression du consentement est implicite car elle découle naturellement de la conduite du sujet qui s'abstient de révoquer sa volonté initiale.

La formalité de l'écrit peut engendrer un effet indésirable sur la conception que le sujet se fait de ses obligations à l'égard des responsables ou des promoteurs de l'expérimentation. Le sujet pourrait percevoir sa signature comme le point final qu'on met à une transaction définitivement engageante. La transaction par laquelle on achète une voiture ou une maison constitue un contrat irrévocable. Il faut éviter que le sujet applique cette logique à l'expérimentation et qu'il entretienne l'impression que sa signature l'engage jusqu'à ce que l'expérimentation soit terminée 83 .

Enfin, même si l'on place le consentement écrit dans un contexte contractuel ${ }^{84}$, la volonté du sujet n'est pas seule en

83. Michel T. Giroux, (2003) 19 Médecine/sciences 1016 à la p. 1017.

84. Didier Lluelles, "Les renouvellements unilatéral et automatique, fondés sur une clause du contrat" (Printemps 2004) 64 R. du B. 151. 
cause $^{85}$. Contrairement au contrat synallagmatique traditionel, le sujet n'a pas le droit de s'engager au-delà d'une limite raisonnable, cest-à-dire lorsque les risques deviennent hors de proportion avec les bénéfices (art. 20 C.c.Q.) : "en matière d'expérimentation, l'inviolabilité de la personne humaine fait prédominer l'intégrité physique et mentale - donc la santé - du sujet sur son autonomie»86. On voit bien ici que le droit reconnaît la prépondérance de l'intégrité personnelle $\mathrm{du}$ sujet sur son autodétermination elle-même, puisque le sujet ne jouit pas d'une totale liberté contractuelle en matière d'expérimentation.

\section{Le formulaire conçu comme un contrat : conséquences pratiques}

Nous reconnaissons, dans le contrat entre le chercheur et le sujet de recherche, certains attributs des contrats en général dont il est question dans le Code civil du Québec ${ }^{87}$.

Tout d'abord, il s'agit d'un contrat synallagmatique dans lequel "les parties s'obligent réciproquement»88. Le sujet de recherche est, en effet, débiteur de certaines obligations : suivre les instructions de l'équipe de recherche, se présenter aux rendezvous, remplir les questionnaires, rester à jeun, répondre honnêtement aux questions, prendre ses médicaments, etc. Enfreindre les instructions risque de biaiser les résultats et de

85. "L'article 20 C.c.Q. édicte qu'une personne peut se soumettre à une expérimentation "pourvu que le risque couru ne soit pas hors de proportion avec le bienfait qu'on peut raisonnablement en espérer". En matière d'expérimentation, le consentement du patient ne suffit donc pas. De fait, ce consentement ne pourra être sollicité, et obtenu, qu'après la prise en considération du rapport risque/bienfait. Ce n'est qu'après cette évaluation que le chercheur pourra envisager de procéder à l'expérimentation et obtenir les consentements des sujets de recherche", Parent c. Maziade, [1998] R.J.Q. 1444 (C.A.) à la p. 1457.

86. Castelli et Cadorette, supra note 57 à la p. 212; Kouri et Nootens, supra note 9.

87. Code civil du Québec, supra note 6 au Livre cinquième, chapitre deuxième, art. 1378 C.c.Q. et ss.

88. Art. 1380 C.c.Q., Lesage-Jarjoura et Philips-Nootens, supra note 39 aux pp. 9-16. 
Le formulaire de consentement

(2007) 37 R.D.U.S. à la recherche : incompatibilité entre

le droit des personnes et le droit des contrats

porter préjudice à la recherche. De son côté, le chercheur doit se présenter aux rendez-vous, respecter le protocole de recherche, s'entourer de personnel compétent, saisir les données de façon rigoureuse, procurer les soins requis, verser les indemnités compensatoires, etc.

Ensuite, même si le Code civil du Québec interdit toute contrepartie financière à l'expérimentation ${ }^{89}$, cela ne signifie pas forcément que le contrat soit à titre parfaitement gratuit ${ }^{90}$. Certes, le contrat peut ne mentionner aucun avantage pécunier ni avantage dans la livraison des soins, mais le sujet éprouve souvent des attentes précises en matière de suivi et d'attentions diverses personnalisées. Il arrive donc souvent que les deux parties retirent un avantage de la recherche : le chercheur cueille des observations et des données, et le sujet reçoit une attention et des soins qu'il ne recevrait peut-être pas en dehors d'un contexte de recherche. Il reçoit parfois même, une indemnité financière non négligeable. Dans ces circonstances, les attentes des patients/sujets pour toutes sortes d'avantages dépassent le don gratuit de leur personne et font en sorte que les contrats de recherche entre eux et les chercheurs ne sont pas toujours à titre gratuit.

Enfin, il s'agit certainement d'un contrat d'adhésion 91 puisque les sujets de recherche n'ont que peu ou pas "le choix quant aux conditions du contrat»92.

Toutes ces caractéristiques typiques des rapports contractuels naissent de l'échange des consentements ${ }^{93}$ entre le chercheur et le sujet de recherche ou son représentant légal ${ }^{94}$. Or, redisons-le, tel qu'il est habituellement libellé, le formulaire de

89. Art. 25 C.c.Q.

90. Art. 1381 C.c.Q.

91. Art. 1379 C.c.Q.; Roy c. Walker, REJB 1997-07492 (C.S.), 18 juin 1997 [Roy c. Walker (C.S.)].

92. Henri Kélada, Précis de droit privé québécois, Québec, Société québécoise d'information juridique, 1986 à la p. 328; Baudouin, Jobin et Vézina, supra note 43 aux p. 66 et ss.

93. Art. 1385 et 1386 C.c.Q.

94. Voir les explications, supra note 56. 
consentement ressemble davantage à une entente contractuelle qu'à une simple autorisation ${ }^{95}$ de donner des soins. On y retrouve en effet, les signatures du chercheur et du sujet de recherche et toutes les modalités d'exécution de la recherche, jusqu'à certaines clauses d'indemnisation.

Un contrat écrit, dans son essence, vise à éviter les malentendus. L'objectif est de rendre la relation contractuelle la plus claire et transparente possible. Il est donc souhaitable de retrouver dans le contrat de nombreuses clauses: clauses sur l'exécution détaillée des obligations, clauses sur l'interprétation du contrat, clauses permettant la gestion de la mésentente ${ }^{96}$, clauses pénales ou d'indemnisation ${ }^{97}$, etc. Il s'agit d'une logique visant à prévenir les conflits ${ }^{98}$ dans laquelle on devrait idéalement stipuler d'avance toutes les modalités de l'entente entre les contractants. On comprend facilement qu'une telle perception du formulaire de consentement viendrait anéantir à leur base les arguments réclamant un formulaire limité à "ce qui est essentiel à l'obtention du consentement»99.

En conséquence, il n’y aurait pas de limites à la prévoyance et on voit mal, si l'on devait suivre en priorité la rationalité contractuelle, sur quelles bases légales ou morales un CÉR

95. Knoppers, supra note 27.

96. On suggère de plus en plus d'insérer une clause d'arbitrage ou de médiation obligatoire dans les contrats. À cet effet, le 3 octobre 2002, l'Institut de médiation et d'arbitrage du Québec a officiellement lancé une campagne de promotion "Servez votre cause, exigez la clause" incitant les contractants à prévoir la médiation ou l'arbitrage obligatoire en cas de conflit" (7 avril 2007) en ligne : IMAQ : <http://www.iamq.org/>. À l'avenir, devra-t-on prévoir ce type de clause dans le formulaire de consentement?

97. Art. 1474 C.c.Q.

98. Capitant, supra note 72 .

99. Lesage-Jarjoura et Philips-Nootens, supra note 39 à la p. 168 : "Il a été établi que le degré de compréhension varie suivant la longueur du texte. Les formules [formulaires de consentement] trop courtes ont pour défaut d'être incomplètes; les formules trop longues rendent la décision douteuse. Les formules ne devraient comprendre que ce qui est essentiel à l'obtention $\mathrm{du}$ consentement, et elles ne remplacent pas les explications verbales". Les auteurs renvoient notamment à P.S. Applebaum, "Assessing Patient's Capacity to Consent to Treatment" (1988) 319 : 25 N. Engl. J. Med. 1635; voir aussi Audy, supra note 82. 
Le formulaire de consentement

(2007) 37 R.D.U.S. à la recherche : incompatibilité entre

le droit des personnes et le droit des contrats

pourrait s'opposer à la multiplication de toutes ces clauses dans la mesure où le consentement aurait été éclairé. De la même façon, les CÉR seraient bien mal équipés pour juger de la légitimité du contenu des clauses du contrat. Sauf pour la présence évidente d'une infraction à l'ordre public, la seule responsabilité du CÉR à l'égard du formulaire reviendrait donc à vérifier que tous les éléments (surtout les risques ${ }^{100}$ ) y soient clairement énoncés. Telle serait, à l'égard des CÉR, la conséquence d'une vision exclusivement contractuelle $\mathrm{du}$ formulaire de consentement. On saisit assez vite que cette logique est inapplicable de manière exclusive et s'oppose même, dans une certaine mesure, au mandat des CÉR dont la mission est "de contribuer à s'assurer que toute la recherche avec des sujets humains se déroule conformément à des principes éthiques»101. En effet, en abandonnant l'évaluation du contenu des formulaires de consentement, les CÉR perdraient le pouvoir de vérification qu'ils exercent présentement sur l'information devant être divulguée aux sujets de recherche. On voit mal ensuite comment ils pourraient s'assurer a priori $\mathrm{du}$ respect de l'autonomie (consentement éclairé) de ces derniers ${ }^{102}$.

100. Weiss c. Solomon, supra note 9. À la page 742 de son jugement, le juge De Blois reproche au CÉR d'avoir approuvé un protocole de recherche ainsi qu'une formule de consentement où tous les risques n'étaient pas énumérés : "en matière de recherche purement expérimentale, le médecin doit révéler tous les risques connus même rares ou éloignés et, à plus forte raison, si ceux-ci sont d'une conséquence grave»; voir aussi : Jean-Louis Baudouin, "L'expérimentation sur les humains : un conflit de valeurs" (1980-81) 26 R.D. McGill 809 à la p. 824.

101. Énoncé de politique des trois Conseils, supra note 10 à la p. 1.1. L'Avis juridique du ministère de la Santé et des Services sociaux établissant les Conditions d'exercice des comités d'éthique de la recherche désignés ou institués par le ministre de la Santé et des Services sociaux en vertu de l'article 21 du Code civil, donne mandat au CÉR "d'approuver les projets de recherche effectués auprès de personnes mineures ou majeures inaptes [...]". Dans le cadre de ce mandat, le CÉR doit: "examiner le mode de sélection des personnes et évaluer les modalités de consentement à la recherche", G.O.Q 1998.I.1039.

102. Julien Cabanac, "L'utilisation des règles juridiques dans l'évaluation du risque/bénéfice par les comités d'éthique de la recherche", dans Christian Hervé et al., dir., Place de la bioéthique en recherche et dans les services cliniques, Paris, Dalloz, 2005, 23. 
Sans la présence d'un soutien juridique provenant du CÉR, les sujets se trouveraient exposés à des abus contractuels. En effet, les rédacteurs pourraient leur imposer des obligations conformes à la loi, mais non conformes à l'éthique. Nous pensons par exemple à une obligation de divulgation généreuse de renseignements personnels et à des limites abusives concernant l'indemnisation en cas de préjudice. Les sujets pourraient aussi se croire liés par des clauses contractuelles non conformes aux lois $\mathrm{du}$ Québec, telles certaines clauses d'exclusion de responsabilité103.

Heureusement, ces dernières années la jurisprudence a régulièrement rappelé la distinction entre la réalité du consentement et la signature du formulaire ${ }^{104}$, insistant sur le devoir d'information des professionnels de la santé105, surtout si le formulaire est d'une lecture difficile.

103. Art. 1474 C.c. Q.: "Une personne [...] ne peut aucunement exclure ou limiter sa responsabilité pour le préjudice corporel ou moral causé à autrui».

104. Cantin-Cloutier c. Gagnon, (C.S.) 200-17-001424-993, 20 nov. 2000, REJB 2000-21212; Roy c. Walker, 16 mai 2000, REJB 2000-18273 (C.A.) [Roy c. Walker (C.A.)]; Jacob c. Roy, 2003BE-482 (C.S.); Jimenez c. Pehr, REJB 2002-32698 (C.S.). "Elles [les formules de consentement] font preuve de l'obtention du consentement certes, mais non pas forcément de l'exécution de l'obligation de renseigner. On devra toujours pouvoir démontrer qu'une information adéquate a été donnée au malade. Un consentement signé n'équivaut pas à un consentement éclairé», Lesage-Jarjoura et PhilipsNootens, supra note 38 à la p. 167; Kouri et Nootens, supra note 9 aux p. 298 et 505; Fay A. Rosovsky, Consent to Treatment : a Practical Guide, $2^{\text {nd }}$ ed., Boston, Little, Brown \& Compagny, 1990 à la p. 35; Gilbert Sharpe, The law and Medicine in Canada, 2nd ed., Toronto, Butterworths, 1987 à la p. 89.

105. Cette distinction est assez claire dans le Code de déontologie des médecins (Code des professions, L.R.Q., c. C-26, art. 87; 2001, c. 78, art. 6) qui sépare les explications du consentement écrit :

"30. Le médecin doit, vis-à-vis des sujets de recherche ou de leur représentant légal, s'assurer :

$1^{\circ}$ que chaque sujet soit informé des objectifs du projet de recherche, des avantages, risques ou inconvénients pour le sujet, des avantages que lui procureraient des soins usuels s'il y a lieu, ainsi que du fait, le cas échéant, que le médecin retirera des gains matériels de l'inscription ou du maintien du sujet dans le projet de recherche; 
Le formulaire de consentement

(2007) 37 R.D.U.S. à la recherche : incompatibilité entre

le droit des personnes et le droit des contrats

Dans l'affaire Roy c. Walker106, le juge Tellier déclare non applicable le formulaire de consentement en raison notamment de sa complexité :

[d]ans la mesure où ce document peut constituer un contrat, le Tribunal n'a aucune hésitation à le considérer comme abusif et à le déclarer non applicable. Ce qui doit primer dans les circonstances, ce n'est pas la formule qui en dit tellement qu'elle ne dit pas grand chose. Ce qui apparaît important, ce sont les témoignages des parties et le dossier-patient ${ }^{107}$.

La Cour d'appel va plus loin et n'examine même pas le formulaire dont la fonction serait simplement de faire état de l'existence du consentement :

Avec égards, il est inutile, en l'espèce, d'évaluer le document auquel l'intimée a souscrit. Il est clair qu'elle a consenti au traitement proposé par son dentiste. La question n'est pas celle-là mais celle de savoir si la signataire a reçu tous les renseignements et informations pertinents pour permettre un consentement éclairé au traitement suggéré108.

En bref, on se retrouve dans une situation où les formulaires de consentement ressemblent de plus en plus à des contrats alors que la rationalité contractuelle (art. 1399 C.c.Q) ne semble, encore, être appliquée ni par la jurisprudence, ni par les CÉR. À l'instar des CÉR, les juges sont sans doute sensibles à l'importance de la protection des personnes et en même temps conscients de l'absurdité d'une vision exclusivement contractuelle dans l'interprétation des formulaires de consentement.

$2^{\circ}$ qu'un consentement libre, éclairé, écrit et révocable en tout temps, soit obtenu de chaque sujet avant le début de sa participation à la recherche ou lors de tout changement significatif au protocole de recherche".

106. Roy c. Walker (C.S.), supra note 91.

107. Ibid., à la note 11 du jugement.

108. Roy c. Walker (C.A.), supra note 104. 


\section{CONCLUSION}

$\mathrm{Au}$ fil du temps, les quelques pages faisant preuve de l'existence du consentement à l'expérimentation se sont transformées en un volumineux document contenant une multitude de renseignements sur les droits et les obligations des parties. À tel point que les CÉR, qui jadis encourageaient l'exhaustivité, doivent maintenant restreindre les ardeurs des rédacteurs, non seulement dans la quantité d'informations, mais dans la teneur de ces informations.

En l'absence de précision expliquant la fonction ou la raison d'être de l'écrit exigé par l'article 24 C.c.Q., comment blâmer une tendance qui chercherait, comme pour n'importe quel autre contrat, à y inclure le plus d'informations possibles? Pourquoi aussi freiner le nombre de clauses informant souvent et engageant parfois les sujets de recherche dans toutes sortes d'obligations dont ils ne pourront pas se plaindre puisqu'ils ont signé le formulaire? S'ils signent, c'est qu'ils acceptent! Par contre, les quelques jugements qui constituent la jurisprudence en ce domaine tendent à rejeter cette mentalité.

De par sa nature, le formulaire de consentement se trouverait-il donc entre deux chaises? S'agit-il d'un contrat d'adhésion ou d'un document d'information? Le premier fait appel à une rationalité contractuelle alors que le second s'inscrit dans le cadre de la protection de la personne physique. 
Le formulaire de consentement

(2007) 37 R.D.U.S. à la recherche : incompatibilité entre

le droit des personnes et le droit des contrats

La première fonction des CÉR est la protection des sujets ${ }^{109}$. Conséquemment, il est naturel qu'ils abordent le contenu du formulaire en ayant comme préoccupation l'inviolabilité de la personne. Les deux rationalités ont des objectifs différents; le droit contractuel pousse à l'exhaustivité de l'écrit, alors que le droit des personnes favorise, certes, une information complète mais en mettant surtout l'accent sur la compréhension des sujets (art. 10 C.c.Q.) laquelle rime le plus souvent avec concision. Du reste, certaines clauses d'indemnisation, d'obligation, d'utilisation des résultats, parfaitement justifiées dans un cadre contractuel sont contestables, principalement par ce qu'elles n'ont pas de lien avec les risques de la recherche. Il faut donc conclure à la nécessité d'un certain ajustement.

Si la forme "contrat" était choisie, il faudrait, afin de mieux protéger les sujets de recherche, être plus explicite et distinguer les informations relatives aux soins, à la prise des données et à leur utilisation, de celles relevant de la relation générale chercheur/sujet (ex. : indemnisation en cas de lésion, clauses

109. Cet objectif figure toujours parmi des premières dispositions des règlements universitaires relatifs à la recherche avec des êtres humains. Le règlement amendant la réglementation de la Loi sur les aliments et drogues D.O.R.S./2001-203, décrit le mandat des CÉR à l'article C. 05.001 : "the principal mandate of which is to approve the initiation of, and conduct periodic reviews of, biomedical research involving human subjects in order to ensure the protection of their rights, safety and well being". Par ailleurs, les règles d'éthiques de Santé Canada définissent le comité d'éthique de la façon suivante à l'article 1.27 : «Organisme indépendant (conseil ou comité d'examen de l'établissement, régional, national ou supranational), composé de professionnels des milieux médical et scientifique et de membres n'appartenant pas à ces milieux, dont la responsabilité consiste à assurer la protection des droits, de la sécurité et du bien-être des sujets humains participant à un essai et à garantir publiquement cette protection, d'une part, en examinant le protocole d'essai, la compétence des investigateurs, les installations ainsi que les méthodes et les documents à utiliser pour obtenir le consentement éclairé des sujets après les avoir informés adéquatement et, d'autre part, en formulant une approbation/opinion favorable à cet égard". Elles prévoient aussi explicitement la priorité des participants sur la science à l'article 2.3 : "Les droits, la sécurité et le bienêtre des sujets ont préséance et doivent l'emporter sur les intérêts de la science et de la société». 
pénales, informations accessoires, etc.). En revanche, si l'on préférait revenir à un document ou feuillet d'information (comme l'intitulent parfois les promoteurs de la recherche) destiné exclusivement à l'éclairage du sujet dans le cadre de l'inviolabilité de sa personne, ce document ne devrait contenir que l'information essentielle à la prise de décision. Par exemple, il ne faudrait plus retrouver ces longues et difficiles pages décrivant dans le menu détail chaque intervention, car la présence de ces descriptions ardues ne facilite pas forcément la compréhension du signataire.

Il est difficile de trouver des critères permettant de distinguer l'essentiel de l'accessoire mais cela n'est pas impossible. Le critère le plus simple est sans doute celui de la longueur du formulaire de consentement; est seule essentielle, l'information qui tient dans 5 à 6 pages. On pourrait y parvenir par des rédactions successives. Le contenu du premier jet serait aussi long qu'on le désirerait. Il y aurait ensuite une succession de rédactions jusqu'à ce que le formulaire atteigne 5 à 6 pages. Dans un tel exercice, les rédactions successives du formulaire seraient toujours plus brèves et répondraient chaque fois à la question de savoir ce qui peut être retiré sans que la qualité du consentement du sujet n'en soit altérée. Chaque information conservée devrait être la plus synthétisée possible afin d'en faciliter la compréhension et l'intégration par le sujet de recherche. 